\title{
Outcomes of preimplantation genetic diagnosis in neurofibromatosis type 1
}

\section{Citation}

Merker, Vanessa L., Timothy P. Murphy, J. Bryan Hughes, Alona Muzikansky, Mark R. Hughes, Irene Souter, and Scott R. Plotkin. 2015. "Outcomes of Preimplantation Genetic Diagnosis in Neurofibromatosis Type 1." Fertility and Sterility 103 (3) (March): 761-768.e1. doi:10.1016/ j.fertnstert.2014.11.021.

\section{Published Version}

doi:10.1016/j.fertnstert.2014.11.021

\section{Permanent link}

http://nrs.harvard.edu/urn-3:HUL.InstRepos:33788495

\section{Terms of Use}

This article was downloaded from Harvard University's DASH repository, and is made available under the terms and conditions applicable to Open Access Policy Articles, as set forth at http:// nrs.harvard.edu/urn-3:HUL.InstRepos:dash.current.terms-of-use\#OAP

\section{Share Your Story}

The Harvard community has made this article openly available.

Please share how this access benefits you. Submit a story.

\section{Accessibility}


Outcomes of Preimplantation Genetic Diagnosis in Neurofibromatosis Type 1

Authors: Vanessa L. Merker, BS, ${ }^{a}$ Timothy P. Murphy ${ }^{\text {a }}$ J. Bryan Hughes, BA ${ }^{\text {b; }}$ Alona Muzikansky, MA' ${ }^{\mathrm{c}}$; Mark R. Hughes, MD, PhD ${ }^{\mathrm{b}}$; Irene Souter, MD ${ }^{\mathrm{d}^{*}}$; and Scott R. Plotkin, $\mathrm{MD}, \mathrm{PhD}^{\mathrm{a}^{*}}$

\author{
Affiliations: \\ St., Yawkey 9E, Boston, MA, USA \\ ${ }^{\mathrm{b}}$ Genesis Genetics, 705 South Main St, Plymouth, MI, USA \\ Boston, MA, USA \\ Center, 55 Fruit St, Yawkey 10A, Boston, MA, USA \\ *These authors should be considered similar in author order. \\ Corresponding author: \\ Scott R. Plotkin, MD, PhD \\ Pappas Center for Neuro-Oncology, Yawkey 9E \\ Massachusetts General Hospital \\ 55 Fruit Street \\ Boston, MA 02114 \\ Fax: 617-643-2591 \\ Telephone: 617-726-3650 \\ Email: splotkin@mgh.harvard.edu
}

${ }^{a}$ Department of Neurology and Cancer Center, Massachusetts General Hospital, 55 Fruit

' Biostatistics Center, Massachusetts General Hospital, 55 Staniford Street, Suite 560,

${ }^{\mathrm{d}}$ Department of Obstetrics and Gynecology, Massachusetts General Hospital Fertility

Additional author emails: vmerker@mgh.harvard.edu; tpmurp14@g.holycross.edu; bryan.hughes@genesisgenetics.org, amuzikansky@mgh.harvard.edu; mark.hughes@genesisgenetics.org; isouter@mgh.harvard.edu

Disclosures: TM received funding from the Alvan T. \& Viola D. Fuller - American Cancer Society Junior Research Fellowship. VM received travel expenses from Genesis Genetics. JBH is employed by and MH has a financial interest in Genesis Genetics, a provider of preimplantation diagnostic services.

Capsule: In a retrospective study of patients with neurofibromatosis 1 undergoing PGD, only $37 \%$ of all biopsied embryos were confirmed to be unaffected by NF1 and at least $27 \%$ of couples achieved a live birth. 


\section{ABSTRACT}

Objective: To examine the effect of patient and facility level factors on the success of pre-implantation genetic diagnosis (PGD) in patients with neurofibromatosis 1 (NF1)

Design: Retrospective review

Setting: Large PGD reference laboratory

Patients: All patients with NF1 referred between June, 2004 and May, 2013

Main Outcome Measures: Embryos' NF1 mutation status and live birth rates

Results: 77 couples underwent 156 PGD cycles during the study period. The average maternal age at time of embryo biopsy was 33.2 years,. The majority of embryos had a day 3 single blastomere biopsy without aneuploidy screening. A diagnosis was obtained for $80 \%$ of biopsied embryos; $20 \%$ of biopsies were non-diagnostic due to technical failures. Diagnosis was more often obtained for embryos of parents with familial disease and for embryos biopsied at centers which referred multiple NF1 cases. Among diagnosed embryos, 483/1060 (46\%) were unaffected by the parental NF1 mutation. $22 / 156(14 \%)$ of cycles had a confirmed live birth; if the observed success rate was applied to cycles with unknown outcomes, 33/156 (21\%) of cycles are expected to have resulted in live birth. In multivariate logistic regression, having a live birth was significantly associated with having more unaffected embryos available for transfer $(\mathrm{OR}=1.33$ per additional embryo, 95\% CI: 1.02-1.72).

Conclusion: Advances in biopsy and diagnostic techniques which increase the number of unaffected embryos may improve live birth rates for patients with NF1. Clinicians should counsel patients about their fertility and reproductive options early, using diseasespecific data to set appropriate expectations for the PGD process. 
PGD Outcomes in NF1

Keywords: PGD, NF1, fertility, pregnancy, in vitro fertilization, genetic counseling 


\section{INTRODUCTION}

Preimplantation genetic testing is a molecular technique in which an embryo generated by in vitro fertilization (IVF) can be tested for a genetic condition prior to uterine transfer.(1) By amplifying and examining genetic material from a cellular biopsy of the embryo, a laboratory can determine which embryos may carry certain genetic abnormalities, and doctors can implant only those embryos shown to be free of these abnormalities.(2) Preimplantation genetic diagnosis (PGD) is used to analyze embryos for specific genetic mutations found in one or both parents, while preimplantation genetic screening (PGS) tests embryos for chromosomal aneuploidies. Preimplantation genetic testing can be viewed as an early form of prenatal diagnosis and offers an alternative for couples who wish to increase their chances of giving birth to a healthy child, but are unwilling to terminate an affected pregnancy.(3)

Since its first application in preventing transmission of X-linked disorders(4) and cystic fibrosis,(5) PGD has become an increasingly popular technique for couples affected by monogenic disorders to reduce their risks of having a similarly affected child. $(5,6)$ In particular, the number of PGD analyses being performed for patients with cancer predispositions syndromes has been steadily increasing over the past decade.(7)

One such cancer predisposition syndrome is neurofibromatosis type 1 (NF1), an autosomal dominant, monogenic disorder that displays full penetrance. NF1 is the most common neurogenetic condition (with a birth prevalence of approximately 1 in $3000),(8,9)$ and the sixth most common monogenic disorder to be tested using PGD.(10) Clinical manifestations of NF1 include multiple benign nerve sheath tumors (neurofibromas), intellectual impairments, and bony abnormalities, and persons with NF1 
have an increased risk of developing cancers such as sarcomas and gliomas.(11)

Comprehensive genetic analysis of the NF1 gene has been available since 2000 (12) and current testing can identify a causative genetic alteration in $>95 \%$ patients who meet established clinical criteria, allowing many couples affected by NF1 the opportunity to pursue PGD.

Currently, there are limited data available about the outcomes of PGD for NF1 patients. Previous publications have documented aggregate outcomes of PGD for multiple monogenic diseases $(10,13,14,15,16)$ and prior NF1-specific reports have been case series of a small number of patients.(17,18,19) For this reason, we retrospectively evaluated the diagnostic and clinical outcomes for couples affected by NF1 who pursued PGD at a large international laboratory that provides PGD services.

\section{MATERIAL AND METHODS:}

We retrospectively reviewed the medical records of an international laboratory providing testing for couples who pursued PGD to reduce the risk of transmitting NF1. We included all couples with NF1 referred to laboratory from the start of NF1 testing until May 2013. This research was reviewed by the Partners Institutional Review Board and determined to be non-human subjects research.

\section{PGD Protocol}

Oocyte stimulation was conducted according to referring IVF center's individual protocols. Embryos were fertilized using intracytoplasmic sperm injection (ICSI) to minimize the risk of subsequent sample contamination from supernumerary sperm 
embedded in the zona pellucida. DNA diagnostic reports from a CLIA-approved laboratory and buccal swab samples from appropriate family members were sent to the laboratory to generate molecular probes via a standardized procedure.

Genetic material was obtained from embryos primarily by cleavage-stage biopsy of a single blastomere on day three of development ( $>95 \%$ of cases). Biopsy procedures for PGS were identical to those used in PGD, and additional biopsies were not required when both procedures were performed. Biopsy samples were transported in individual tubes in a lysis buffer by courier to the laboratory for PGD analysis prior to implantation of embryos.

Before 2010, PGD analysis began with direct amplification of the DNA from the diploid cell(s). The cells were lysed and a PCR reaction was performed amplifying the genetic region in question from each cell. An outer amplification reaction and an inner amplification reaction were performed to isolate the mutated region in the gene. All patient samples received after 2010 were subjected to whole-genome amplification (WGA) protocols, rather than direct amplification. Samples were amplified using the BlueGnome SurePlex system. Biopsy samples underwent genomic amplification followed by locus-specific standard PCR of 2 ul aliquots. Six fully-informative, NF1flanking genomic markers were tested independently to determine the haplotype of each sample; this was done to evaluate the remote possibility of an intragenic recombination. After standard PCR of each locus, the amplified DNA was analyzed on an Applied Biosystems 3300 capillary electrophoresis instrument, with independent genotype analysis of each allele. Genotypes were then compared to the haplotype of the gamete providers to determine the $\mathrm{NFI}$ status of each embryo. 
For samples also undergoing PGS, amplified DNA was labeled and hybridized onto Illumina 24sure BAC microarrays. Microarrays were washed and scanned, and single channel images were imported into Illumina software. Fixed algorithms automatically determined whole chromosome gains and losses, while segmental chromosome deletions or duplications larger than $10 \mathrm{Mbp}$ were examined manually. Reports from NF1 PGD analysis, and if applicable, PGS analysis, were issued to the referring IVF center within 48 hours. Pregnancy test and birth outcomes were voluntarily reported by IVF centers back to the diagnostic laboratory.

\section{Data Collection}

\section{Demographics}

We collected demographic data on affected partner's inheritance pattern for NF1 (sporadic or familial), maternal and paternal age, gender of the affected partner, history of infertility, and prior use of IVF. Referring IVF centers were categorized based on number of NF1 cases completed at this PGD laboratory by May, 2013 (single referral vs. multiple referrals) and on practice type (academic vs. non-academic affiliation).

\section{Embryo data collection}

We collected data on the number, quality, and stage (cleavage stage vs. trophectoderm) of all biopsied embryos within each IVF cycle for NF1 patients. Embryo quality was rated by referring centers using a three-point, four-point, or five-point scale. For consistency in our analyses, four- and five-point ratings were rescaled to a three-point scale (good, fair, and poor). 
Additional laboratory data retrieved included the NF1 status of each biopsied cell, and the diagnosis of aneuploidy for any cycles also being tested with PGS. Embryos that displayed normal $N F 1$ alleles were classified as unaffected, while embryos that displayed the mutant parental allele were considered affected. If the laboratory report indicated amplification failure of the probe or no molecular signal, the outcome was classified as "insufficient data". Embryos were classified as "inconclusive results" if the laboratory report indicated allele drop-out, weak amplification, conflicting marker data, recombination, incomplete analysis, or multiple parental alleles observed. Outcomes of PGS were classified as euploid, aneuploid, or no signal.

\section{Clinical outcomes}

We collected all available data on clinical outcomes for all PGD cycles, including pregnancy test results and occurrence of a live birth. While clinical outcomes were not routinely collected by the reference laboratory (since all clinical care took place at the referring IVF center), many IVF centers voluntarily reported outcome data back to the laboratory. Cycles which produced only affected and/or aneuploid embryos were assumed to not have led to transfer or live birth. We also assumed no live birth occurred if a cycle resulted in a negative pregnancy test or the couple began a new PGD cycle within the following nine months. Reasons for no live birth after positive pregnancy test were not available, but would include biochemical pregnancy, spontaneous abortions, and elective terminations. 


\section{Statistical analysis}

$\underline{\text { Factors related to ability to obtain a molecular diagnosis }}$

Descriptive statistics of the patient cohort and embryo characteristics were tabulated. If two biopsies were performed on the same embryo, the most informative diagnosis was used for analysis (i.e status as affected/unaffected rather than insufficient data or inconclusive result). Chi-square analyses were used to test for an association between the ability to obtain a molecular diagnosis on an embryo (as either affected or unaffected with NF1) with the following variables: embryo quality rating, familial or sporadic mutation, number of NF1 referrals from IVF center, IVF practice type, and use of whole-gene amplification. We excluded from these analyses seven samples which could not be analyzed due to breakage during shipping.

\section{Factors associated with PGD resulting in live birth}

Descriptive statistics were generated for the number of pregnancies and live births observed. We also generated estimated pregnancy and birth rates, which took into account the likely outcomes of cycles with missing data in order to predict what the true pregnancy and birth rates may have been in our cohort. To derive the estimated number of unobserved pregnancies, we multiplied the observed pregnancy rate in women with an embryo transfer by the number of cycles with unknown pregnancy outcome. To derive the estimated number of unobserved live births, we multiplied the observed birth rate in pregnant women by the number of observed and unobserved pregnancies with unknown outcome. 
We performed logistic regression analysis to determine which factors correlated with live birth. Dependent variables included number of unaffected embryos produced, maternal age, number of NF1 referrals from the IVF center to this diagnostic laboratory, and time elapsed since the first PGD analysis was performed (in months). Time elapsed since the first PGD analysis was included in our statistical analysis to control for improvements in PGD and IVF technique over time. We excluded from this analysis 24 cycles which did not produce any unaffected embryos, 1 cycle for which embryo transfer was known not to have occurred, and 4 cycles with incomplete data on number of unaffected embryos produced.

All statistical analysis was conducted using SAS version 9.2.

\section{RESULTS}

\section{Patient demographics and clinic characteristics}

From June 2004, to May 2013, 81 couples affected by NF1 began the PGD process, and 77 couples completed at least one PGD cycles. Three couples did not undergo an IVF cycle because molecular probes could not be generated for PGD analysis, and one couple underwent an IVF cycle but did not undergo PGD analysis because no embryos of sufficient quality for biopsy were produced. A total of 156 PGD cycles were completed, with a median number of 2 cycles per couple (range, 1 - 9 cycles). Thirtythree couples $(43 \%)$ completed one cycle, $24(31 \%)$ completed two cycles, $12(16 \%)$ completed three cycles, and $8(10 \%)$ completed four or more cycles. 42 cycles $(27 \%)$ were performed in women with advanced maternal age (age $\geq 35$ years). 
The affected partner was female in 51 couples $(66 \%)$. There were 48 sporadic NF1 patients (62\%) and 23 familial patients (30\%); the inheritance pattern was unknown or not reported in 6 patients (8\%). Fourteen (18\%) couples had a history of infertility and $4(5 \%)$ had previously undergone IVF. The average maternal and paternal age at the time of embryo biopsy was 33.2 years (range, 23.7-43.1 years) and 35.6 years (range, 25.857.5 years), respectively.

Couples were referred from 62 IVF centers from 18 U.S. states and 6 non-U.S. countries (Table 1). 51 centers referred one couple for NF1 PGD, and 11 centers referred more than 1 couple. The clinic with the highest number of NF 1 cases referred eight patients for PGD, seven of whom completed a PGD cycle. 14 IVF centers were academically affiliated, and 47 were independent IVF centers. Throughout the nine years of data collection, the use of PGD by couples affected by NF1 steadily increased from 3 in 2004 to a peak of 38 cycles in 2010.

\section{PGD Cycle Outcomes}

Over 156 PGD cycles, 1356 embryos were biopsied (Figure 1). The median number of embryos biopsied per cycle was 8 (range, 1 to 40 embryos) for all women and was 6 (range, 1 to 13 embryos) for women of advanced maternal age ( $\geq 35$ years). 34 embryos (2.5\%) were biopsied twice. $88 \%$ of biopsied embryos (1193/1356) were fresh, and $12 \%$ of biopsied embryos (163/1356) were frozen. By IVF center self-report, $23 \%$ of biopsied cells were of good quality, $58 \%$ were of fair quality, $8 \%$ were of poor quality, and $11 \%$ were not rated. Whole-genome amplification was used to analyze 782 embryos (within 90 cycles) and was not used in 574 embryos (within 66 cycles). 
PGD outcomes were available for 1322/1356 (97\%) of the biopsied embryos and a clear molecular diagnosis was obtained for 1060/1322 (80\%) embryos. Insufficient molecular data was observed in 161/1322 (12\%) embryos and 101/1322 (8\%) embryos produced inconclusive results. Of the embryos with a molecular diagnosis, 483/1060 (46\%) were unaffected with NF1. The number of unaffected embryos produced per PGD cycle ranged from 0 to 18 , with a median number of 3 unaffected embryos per cycle. $135 / 156(87 \%)$ cycles produced at least one unaffected embryo.

Definitive diagnoses were more likely to be obtained for embryos of parents with familial NF1 inheritance (84\%) compared to embryos from parents with sporadic NF1 $(76 \%)\left(\chi^{2}=12.1, p<0.001\right)$. Diagnoses were also more likely to be obtained for embryos biopsied at centers with multiple NF1 referrals (83\%) compared to embryos biopsied at centers with a single NF1 referral $(77 \%)\left[\chi^{2}=6.2, \mathrm{p}=0.01\right]$. The ability to obtain a molecular diagnosis was not associated with embryo quality rating ( $\mathrm{p}=0.46$ ), academic affiliation of the referring medical center $(\mathrm{p}=0.18)$, or use of whole-gene amplification in the probe development process $(\mathrm{p}=0.98)$.

\section{PGS cycle outcomes}

Ten couples completing a PGD cycle also underwent PGS in a total of 15 cycles. The average maternal age for patients completing a cycle with PGS was 35.5 years (range, 29.9-42.0 years). PGS was done in addition to PGD on 114 embryos. Of these, 44/114 (39\%) were euploid, 56/114 (49\%) displayed some form of aneuploidy, and 14/114 (12\%) did not display a signal. 26 embryos unaffected by NF1 were not transferred due to the presence of aneuploidy. In 3 cycles, even though embryos without 
NF1 were produced, no embryos were available for transfer due to aneuploidies found during PGS.

\section{Pregnancy and birth outcomes}

132/156 (85\%) cycles had at least one embryo suitable for transfer (Figure 2). Pregnancy test data were available for 80/132 (61\%) cycles with at least one embryo suitable for transfer. Of the cycles with pregnancy test data, $41 / 80(51 \%)$ resulted in a positive serum pregnancy test. In 22 cycles, these pregnancies resulted in a live birth. Multiple births (twin or triplet) accounted for $23 \%$ of the live births and a total of 28 infants were born to 21 different couples. No live birth occurred in 13/41 (32\%) cycles with a positive pregnancy test, and there were no birth outcome available for the remaining 6/41 (15\%) cycles.

Overall, 21/77 (27\%) of couples undergoing at least one IVF/PGD cycle achieved a live birth. Among couples that successfully had a live birth, the median number of cycles to achieve a live birth was 2 (range, 1-5 cycles). Of these couples, 19/21 (90\%) were successful within their first two cycles of PGD. By applying the observed pregnancy and birth rates to cycles for which an outcome was unknown, we estimate that 57/156 (37\%) of cycles resulted in a positive pregnancy test, and that 33/156 (21\%) of cycles resulted in a live birth (Table 2).

In multivariate logistic regression, having a higher number of unaffected embryos available for transfer was significantly associated with having a live birth after a given PGD cycle $(\mathrm{p}=0.03, \mathrm{OR}=1.33$ per additional embryo, 95\% CI: $1.02-1.72)$. Maternal age, 
number of center referrals, and months since the first PGD analysis was performed were not significantly associated with birth outcome.

\section{Reasons for failure of PGD technology}

4/81(5\%) couples affected by NF1 who wished to pursue PGD were not able to. Molecular probes could not be designed for three couples due to the specific nature of their de novo mutations. When attempting to develop molecular probes, it was found that each patient's specific mutation in the NFl gene made it nearly identical to another area of DNA (called a pseudogene). During PCR, both the NF1 gene and the pseudogene were amplified (known as pseudogene bleed-through), which can lead to embryos without the parental mutation presenting as false positives. Without genetic samples available from other affected family members, the risk of false positive was deemed unacceptably high to continue PGD. Additionally, for one couple, no embryos of sufficient quality to biopsy were produced in the only IVF cycle undertaken.

\section{DISCUSSION:}

In this study, we reviewed the records of one of the largest diagnostic laboratories in the United States to evaluate the outcomes of pre-implantation genetic diagnosis for patients affected by NF1. This data provides valuable disease-specific information for counseling couples about the potential benefit of using IVF and PGD to reduce the risk of transmitting NF1 to their offspring. The diagnostic laboratory was able to create molecular probes for most NF1 couples (95\%). Of 1322 biopsied embryos, 483 (37\%) were determined to be unaffected by the parental NF1 mutation. We estimate that $37 \%$ 
of IVF/PGD cycles for NF1 result in a positive pregnancy test, and that $21 \%$ of all cycles result in a live birth. At least $27 \%$ of couples eventually achieved at least one live birth during treatment.

Analysis of disease-specific outcomes after PGD is increasingly important as the scope of reproductive counseling spreads beyond women and men affected by infertility to include patients with genetic abnormalities. In an age where the cost of genome sequencing is dramatically dropping, PGD is becoming available to more patient populations. Between 1997 and 2007, parents used PGD in more than 10,000 IVF cycles to prevent the transmission of a known genetic disorder to a child.(7) NF1 was the third most common autosomal dominant disorder analyzed among centers reporting to the European Society of Human Reproduction and Embryology (ESHRE) consortium in 2008.(10) For this reason, analysis of laboratory and clinical outcomes after PGD in patients with NF is needed to appropriately advise patients with NF1 about their reproductive options.

In our paper, $80 \%$ of biopsied embryos could be classified as affected or unaffected by the parental NF1 mutation. This figure is slightly below the average figure observed between 1997 and 2007 for two other autosomal dominant disorders: $86.6 \%$ for myotonic dystrophy type 1 and $87.8 \%$ of Huntington's disease.(7) Given that the ability to identify the presence or absence of mutations for all monogenic disorders during PGD has improved from $83 \%$ in 1997 to $90 \%$ in 2007 , it is likely that a higher percentage of embryos from couples affected by NF1 are able to be diagnosed today.(7)

Definitive diagnoses were significantly more likely to be obtained for embryos from parents with familial NF1 inheritance compared to embryos from parents with 
sporadic NF1 (i.e. parent was the first in their family to have an NF1 mutation). In familial cases, genetic material could be solicited from additional family members, allowing for more sensitive probe preparation that allowed a greater diagnosis success rate. Diagnoses were also more likely to be obtained for embryos biopsied at centers with multiple NF1 referrals compared to embryos biopsied at centers with a single referral. This difference highlights the importance of consulting medical professionals experienced in PGD for the retrieval and biopsy of embryos.

$46 \%$ of embryos which were able to be diagnosed were unaffected with the parental NF1 mutation. Because NF1 is autosomal dominant, it is expected that an average of $50 \%$ of embryos would be unaffected by the parental mutation. However, in both our series and in prior analysis of ESHRE data on muscular dystrophy 1 (43.4\%) and Huntington's disease (43.9\%)(10), there were fewer unaffected embryos than expected. It is possible that technical difficulties in amplification and analysis are more frequent in unaffected samples, and that future improvements in laboratory analysis will increase the proportion of unaffected embryos observed.

The proportion of PGD cycles that resulted in a live birth in our sample was lower than the proportion of successful IVF cycles reported by the Society for Assisted Reproductive Technology. Given that many patients with NF1 and their partners do not have a history of infertility, patients may be surprised to learn that their reproductive chances are not equal to or even greater than other IVF couples (almost all of whom have experienced infertility). If so, education regarding the implications of selecting embryos based on mutation status rather than morphology may be warranted. 
While couples affected by NF1 pursuing PGD have lower success rates than couples with infertility only, analysis of clinical outcomes showed that a similar number of women in our cohort achieved a positive pregnancy test and live birth compared to women with other autosomal dominant and X-linked disorders (Supplemental Table 1). As expected, the pregnancy and birth rates for couples with NF1 and other autosomal dominant disorders are lower than those for autosomal recessive disorders(10), which are expected to have an average of $75 \%$ of embryos unaffected and available for transfer as opposed to $50 \%$. Thus, previously published data on PGD that has been aggregated across both dominant and recessive disorders will overestimate the success rate of PGD in NF1, and should not be used for comparison to our results.

While the majority of embryos in our study were biopsied at the cleavage-stage, recent evidence suggest that higher live birth rates may be achieved with blastocyst stage biopsy of the trophectoderm.(20,21) In a randomized controlled trial (2013), the adverse effect of cleavage stage biopsy compared to blastocyst biopsy was equivalent to two of every five reproductively competent embryos becoming incapable of sustained implantation.(20,22) As such, increased adoption of blastocyst biopsy could lead to even higher live birth rates in NF1 couples attempting PGD in the future.

In multivariate analysis, the only factor significantly associated with having a live birth was having a larger number of unaffected embryos available for transfer (OR 1.33, [95\% CI, $1.03-1.72]$. This result is unsurprising, given that the more embryos to choose from for implantation, the higher likelihood there will be an optimally developing embryo available. Our results agree with that from Grace et al (2006), who found that 
only significant factor affecting live birth outcome was presence of two or more embryos genetically suitable for transfer.(23)

Our data may be useful in future studies to calculate the cost-effectiveness of PGD in preventing transmission of NF1 to future generations. The average cost of an IVF cycle in the US is between $\$ 12,400$ and $\$ 13,775 .(24,25)$ In addition, ICSI costs an additional $\$ 1500$ per IVF cycle, and PGD costs between $\$ 2500-\$ 5000$ per cycle.(26) Given that the majority of couples in our study who achieved a live birth did so within the first 2 cycles, the direct medical costs of PGD compared to a natural birth are between $\$ 33,000$ and $\$ 40,000$. Comparisons of this figure to the lifetime costs of caring for a child with NF1 will likely reveal a significant savings to insurance companies (at least in the United States) from covering PGD and associated IVF expenses. Future costeffectiveness analyses are imperative to investigate the impact of PGD on the care of NF1 and other genetic disorders.

There were limitations to our study due to its retrospective nature. There was missing data regarding pregnancy test and birth outcomes, and no data available on clinical pregnancy rates. For this reason, we report the possible range of outcomes possible (from the number of births actually observed, to the highest possible number of births if all missing data was positive). In addition, our analysis spanned from 2004 to 2013, and while timing was not significant in our analysis of birth outcomes, our results may not reflect the outcomes that are obtained in current practice. Current use of blastocyst-stage (rather than cleavage-stage) biopsy in particular may result in more favorable outcomes than those reported in this series. 
While having all cases from a single reference laboratory meant that probe preparation and analysis protocols were identical, there may be many differences between referring IVF centers that would affect live birth rates, including ovarian stimulation protocols. Similarly, our classification of centers based on number of NF1 case referrals is a crude marker of a center's volume of PGD cases. We recognize that centers that referred only one NF1 case to this diagnostic laboratory may have referred other cases to other laboratories, and the analysis of centers may be confounded by other systematic differences between the two groups. For this reason, our finding that centers with more case referrals have higher success rates should be validated using stricter criteria. Finally, while embryo quality rating was transformed to a single numerical scale from primary data, the ratings were determined by each referring IVF center. This lack of standardization may have prevented us from recognizing an association between embryo quality and the ability to obtain a diagnosis on the embryo biopsy.

\section{CONCLUSIONS:}

For many patients with NF1, PGD is an attractive option for starting a family. PGD is a useful for couples who want to avoid passing NF1 to the next generation, and are not amenable to alternative methods of prevention (such as prenatal diagnosis followed by elective termination or use of donor gametes). While less than half of patients in our dataset achieved a live birth, strategies that increase the number of unaffected embryos produced and identified in each cycle could improve live birth rates in the future. Identification of the optimal timing of embryo biopsy as well as laboratory 
improvements that decrease the rate of non-informative biopsy results should be especially helpful.

In addition, medical providers and genetic counselors should discuss PGD and with couples early in the care process, so they have time to consider their reproductive preferences before starting a family. While overall pregnancy and birth rates were similar to other autosomal dominant disorders, our analysis revealed several factors that differ between NF1 and other disorders, as well as within NF1 patients. Clinicians can use this disease-specific data to guide their discussion with patients to provide more tailored advice and to set realistic expectations of the PGD process.

\section{ACKNOWLEDGEMENTS:}

We thank John Kitchen, PhD, for his helpful comments on preimplantation diagnosis laboratory techniques. 
PGD Outcomes for NF1

\section{Reference List}

1. Brezina PR, Brezina DS, Kearns WG. Preimplantation genetic testing. BMJ 2012;345:e5908.

2. Hardy KHandyside AH. Biopsy of cleavage stage human embryos and diagnosis of single gene defects by DNA amplification. Arch Pathol Lab Med 1992;116:388-392.

3. Sermon K, Van SA, Liebaers I. Preimplantation genetic diagnosis. Lancet 2004;363:16331641.

4. Handyside AH, Kontogianni EH, Hardy K, Winston RM. Pregnancies from biopsied human preimplantation embryos sexed by $\mathrm{Y}$-specific DNA amplification. Nature 1990;344:768-770.

5. Handyside AH, Lesko JG, Tarin JJ, Winston RM, Hughes MR. Birth of a normal girl after in vitro fertilization and preimplantation diagnostic testing for cystic fibrosis. $\mathrm{N}$ Engl J Med 1992;327:905-909.

6. Blaszczyk A, Tang YX, Dietz HC, Adler A, Berkeley AS, Krey LC, Grifo JA. Preimplantation genetic diagnosis of human embryos for Marfan's syndrome. J Assist Reprod Genet 1998;15:281-284.

7. Harper JC, Wilton L, Traeger-Synodinos J, Goossens V, Moutou C, SenGupta SB, Pehlivan BT, Renwick P, De RM, Geraedts JP, Harton G. The ESHRE PGD Consortium: 10 years of data collection. Hum Reprod Update 2012;18:234-247.

8. Friedman JM. Epidemiology of neurofibromatosis type 1. Am J Med Genet 1999;89:1-6.

9. Huson SM, Harper PS, Compston DA. Von Recklinghausen neurofibromatosis. A clinical and population study in south-east Wales. Brain 1988;111 ( Pt 6):1355-1381.

10. Goossens V, Traeger-Synodinos J, Coonen E, De RM, Moutou C, Pehlivan T, DerksSmeets IA, Harton G. ESHRE PGD Consortium data collection XI: cycles from January to December 2008 with pregnancy follow-up to October 2009. Hum Reprod 2012;27:1887-1911.

11. NIH Consensus Conference. Neurofibromatosis. Conference statement. National Institutes of Health Consensus Development Conference. Arch Neurol 1988;45:575-578.

12. Messiaen LM, Callens T, Mortier G, Beysen D, Vandenbroucke I, Van Roy N, Speleman F, Paepe AD. Exhaustive mutation analysis of the NF1 gene allows identification of $95 \%$ of mutations and reveals a high frequency of unusual splicing defects. Hum Mutat 2000;15:541-555.

13. Gutierrez-Mateo C, Sanchez-Garcia JF, Fischer J, Tormasi S, Cohen J, Munne S, Wells D. Preimplantation genetic diagnosis of single-gene disorders: experience with more 
than 200 cycles conducted by a reference laboratory in the United States. Fertil Steril 2009;92:1544-1556.

14. Feyereisen E, Steffann J, Romana S, Lelorc'h M, Ray P, Kerbrat V, Tachdjian G, Frydman R, Frydman N. Five years' experience of preimplantation genetic diagnosis in the Parisian Center: outcome of the first 441 started cycles. Fertil Steril 2007;87:60-73.

15. Grifo J, Talebian S, Keegan D, Krey L, Adler A, Berkeley A. Ten-year experience with preimplantation genetic diagnosis (PGD) at the New York University School of Medicine Fertility Center. Fertil Steril 2007;88:978-981.

16. Vandervors M, Staessen C, Sermon K, De VA, van d, V, Van AE, Bonduelle M, Vanderfaellie A, Lissens W, Tournaye H, Devroey P, Van SA, Liebaers I. The Brussels' experience of more than 5 years of clinical preimplantation genetic diagnosis. Hum Reprod Update 2000;6:364-373.

17. Chen YL, Hung CC, Lin SY, Fang MY, Tsai YY, Chang LJ, Lee CN, Su YN, Chen SU, Yang YS. Successful application of the strategy of blastocyst biopsy, vitrification, whole genome amplification, and thawed embryo transfer for preimplantation genetic diagnosis of neurofibromatosis type 1. Taiwan J Obstet Gynecol 2011;50:74-78.

18. Spits C, De RM, Van RN, Joris H, Verpoest W, Lissens W, Devroey P, Van SA, Liebaers I, Sermon K. Preimplantation genetic diagnosis for neurofibromatosis type 1. Mol Hum Reprod 2005;11:381-387.

19. Verlinsky Y, Rechitsky S, Verlinsky O, Chistokhina A, Sharapova T, Masciangelo C, Levy M, Kaplan B, Lederer K, Kuliev A. Preimplantation diagnosis for neurofibromatosis. Reprod Biomed Online 2002;4:218-222.

20. Scott RT, Jr., Upham KM, Forman EJ, Zhao T, Treff NR. Cleavage-stage biopsy significantly impairs human embryonic implantation potential while blastocyst biopsy does not: a randomized and paired clinical trial. Fertil Steril 2013;100:624630.

21. Papanikolaou EG, Kolibianakis EM, Tournaye H, Venetis CA, Fatemi H, Tarlatzis B, Devroey P. Live birth rates after transfer of equal number of blastocysts or cleavage-stage embryos in IVF. A systematic review and meta-analysis. Hum Reprod 2008;23:91-99.

22. Scott KL, Hong KH, Scott RT, Jr. Selecting the optimal time to perform biopsy for preimplantation genetic testing. Fertil Steril 2013;100:608-614.

23. Grace J, El-Toukhy T, Scriven P, Ogilvie C, Pickering S, Lashwood A, Flinter F, Khalaf Y, Braude $\mathrm{P}$. Three hundred and thirty cycles of preimplantation genetic diagnosis for serious genetic disease: clinical considerations affecting outcome. BJOG 2006;113:1393-1401. 
24. American Society of Reproductive Medicine. Q06: Is in vitro fertilization expensive? 2013. Available at http://www.reproductivefacts.org/detail.aspx?id=3023. Accessed August 22, 2013.

25. Parikh FR. Affordable in vitro fertilization. Fertil Steril 2013;100:328-329.

26. Galpern, E. Assisted reproductive technologies: overview and persepctive using a reproductive justice framework. 2007. Available at http://www.geneticsandsociety.org/downloads/ART.pdf. Accessed August 22, 2013. 


\section{Figures}

Figure 1: Diagnostic outcomes of PGD for neurofibromatosis 1.

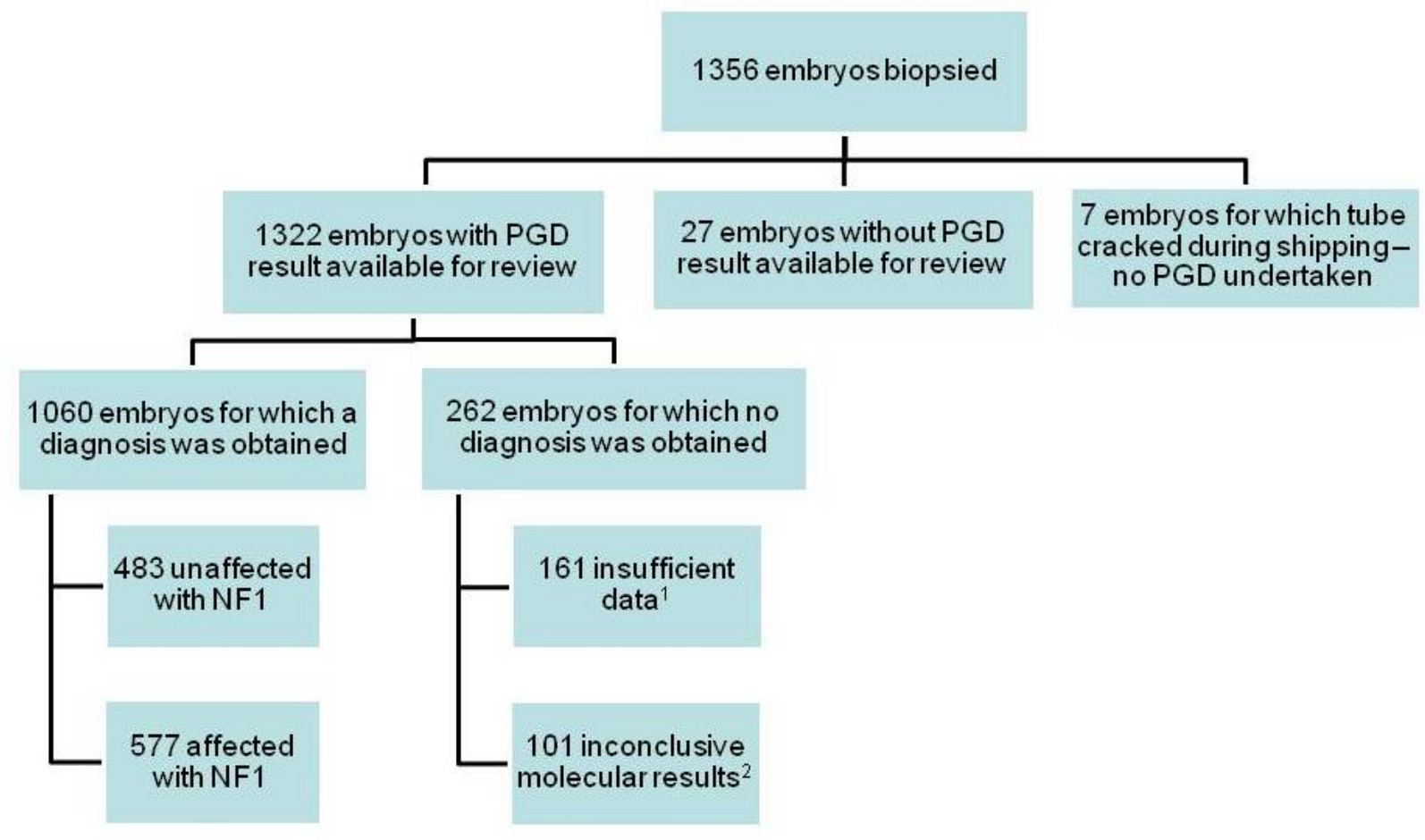

${ }^{1}$ Includes probe amplification failure or no molecular signal

${ }^{2}$ Includes allele drop-out, conflicting marker data, multiple parental alleles observed, or recombination 
Figure 2: Clinical outcomes after PGD for neurofibromatosis 1

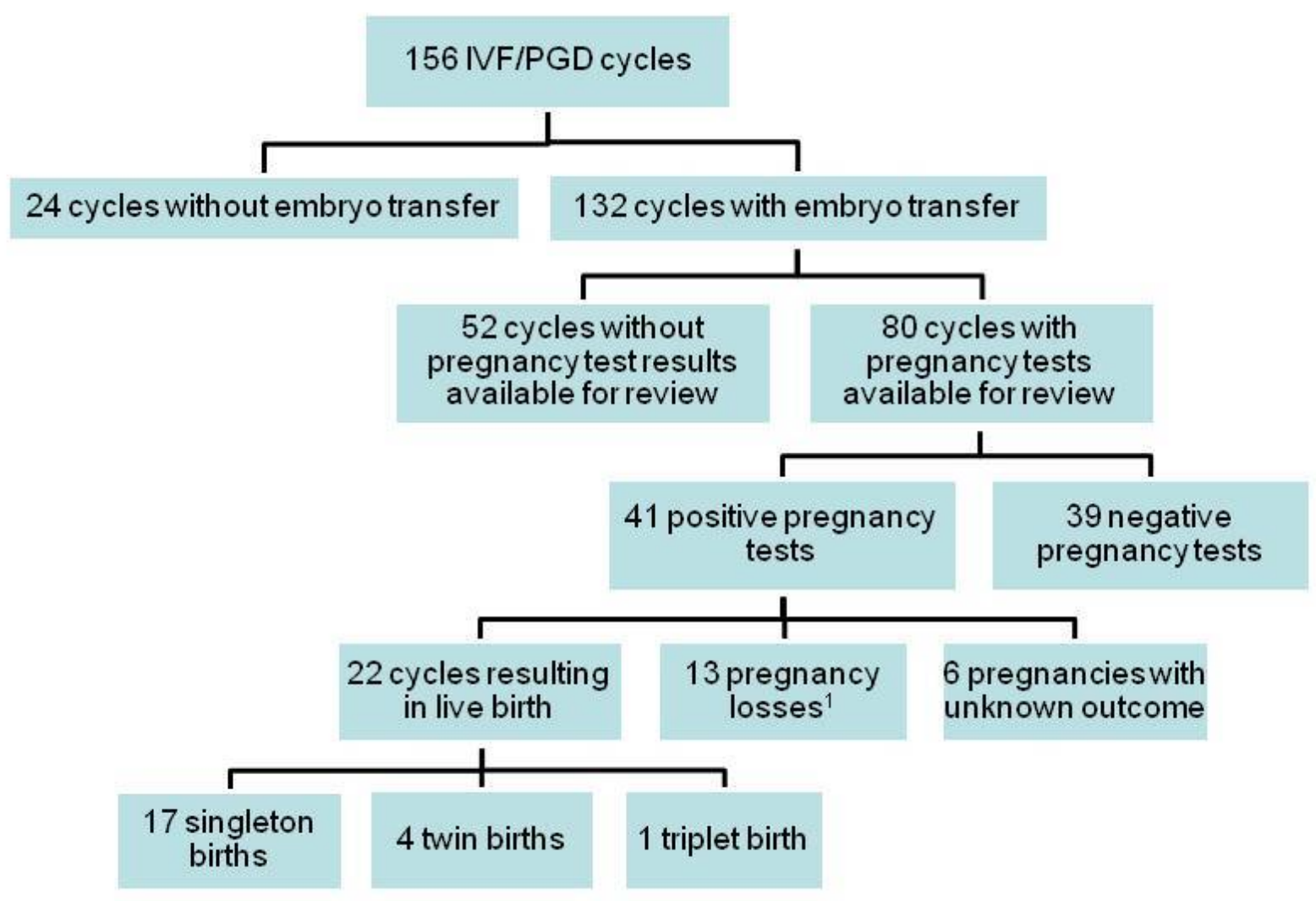

${ }^{1}$ Includes biochemical pregnancy, spontaneous abortion, and/or elective termination 
Table 1. Patient Demographics

\begin{tabular}{|l|l|}
\hline & N (\%) \\
\hline Couples completing PGD analysis & 77 \\
\hline Maternal age (mean \pm SD), years (range) & $33.2 \pm 4.2(23.7-43.1)$ \\
Paternal age (mean \pm SD), years (range) & $35.6 \pm 6.0(25.8-57.5)$ \\
\hline Advanced Maternal Age & \\
Mothers of advanced maternal age ( $>35$ years) & $21(27 \%)$ \\
Mothers not of advanced maternal age & $56(73 \%)$ \\
\hline Affected Partner & \\
Mother & $51(66 \%)$ \\
Father & $26(34 \%)$ \\
\hline Inheritance Pattern & \\
Sporadic & $48(62 \%)$ \\
Familial & $23(30 \%)$ \\
Unknown, not reported & $6(8 \%)$ \\
\hline History of infertility & \\
Present & $14(18 \%)$ \\
Male factor & 8 \\
Idiopathic & 3 \\
Anovulatory & 1 \\
Endometriosis & 1 \\
Other & 1 \\
None & $49(64 \%)$ \\
Unknown & $14(18 \%)$ \\
Prior IVF & $4(5 \%)$ \\
For infertility & 1 \\
For PGD & 1 \\
Unknown reason & 2 \\
None & $12(16 \%)$ \\
Unknown & \\
\hline History of IVF & \\
Pno & \\
\hline
\end{tabular}


Table 2. Clinical outcomes of IVF/PGD cycles for NF1

\begin{tabular}{|l|l|l|}
\hline & Observed & Estimated \\
\hline Number of cycles with embryo biopsy & 156 & 156 \\
Cycles resulting in positive pregnancy test & $41(26 \%)$ & $57(37 \%)$ \\
Cycles resulting in live birth & $22(14 \%)$ & $33(21 \%)$ \\
Number of cycles with embryo transfer & 132 & 132 \\
Cycles resulting in positive pregnancy test & $41(31 \%)$ & $57(43 \%)$ \\
Cycles resulting in live birth & $22(17 \%)$ & $33(25 \%)$ \\
Percentage of live births with twins or triplets & $5(23 \%)$ & $7(21 \%)$ \\
\hline
\end{tabular}


Supplemental Table 1. Comparison of NF1 pregnancy outcomes after PGD (this manuscript) with published data from the European Society of Human Reproduction and Embryology (Goossens et al, 2012).

\begin{tabular}{|c|c|c|c|c|c|c|}
\hline \multirow{2}{*}{$\begin{array}{l}\text { Inheritance Type } \\
\text { Condition studied }\end{array}$} & \multicolumn{4}{|c|}{ Autosomal Dominant } & \multirow{2}{*}{$\begin{array}{l}\text { Autosomal } \\
\text { Recessive } \\
\text { Multiple } \\
\text { disorders }\end{array}$} & \multirow{2}{*}{$\begin{array}{l}\text { X-linked } \\
\text { Multiple } \\
\text { disorders }\end{array}$} \\
\hline & $\mathrm{NF} 1^{1}$ & $\begin{array}{l}\text { Myotonic } \\
\text { dystrophy } \\
\text { type } 1\end{array}$ & $\begin{array}{l}\text { Huntington's } \\
\text { Disease }\end{array}$ & $\begin{array}{l}\text { Multiple } \\
\text { disorders }^{2}\end{array}$ & & \\
\hline Time Period & $2004-2013$ & $1997-2007$ & $1997-2007$ & 2008 & 2008 & 2008 \\
\hline Mean maternal age (years) & 33.2 & 32 & 31 & 34 & 34 & 33 \\
\hline Number of cycles w/ biopsy & 156 & 552 & 516 & 543 & 401 & 223 \\
\hline Positive Pregnancy Test & $26-37 \%$ & $26 \%$ & $28 \%$ & $28 \%$ & $40 \%$ & $28 \%$ \\
\hline Clinical Pregnancy & --- & $19 \%$ & $22 \%$ & $21 \%$ & $30 \%$ & $27 \%$ \\
\hline Live birth & $14-21 \%^{1}$ & ---- & --- & $18 \%-19 \%$ & $24 \%-29 \%$ & $19 \%$ \\
\hline $\begin{array}{l}\text { Number of cycles w/ } \\
\text { embryo transfer }\end{array}$ & 132 & 445 & 421 & 423 & 361 & 171 \\
\hline Positive Pregnancy Test & $31-43 \%$ & $32 \%$ & $34 \%$ & $35 \%$ & $45 \%$ & $36 \%$ \\
\hline Clinical Pregnancy & --- & $24 \%$ & $26 \%$ & $27 \%$ & $33 \%$ & $36 \%$ \\
\hline Live birth & $17-25 \%$ & --- & --- & $23-25 \%$ & $27 \%-32 \%$ & $25 \%$ \\
\hline
\end{tabular}

\footnotetext{
${ }^{1}$ Ranges represent observed and estimated results in the current study.
}

${ }^{2}$ Ranges represent lower and upper limits of possible results, given the amount of missing data. 